\title{
Large Extramedullary Plasmacytoma in Oral Cavity in a patient with Lympho plasmacytic Non-Hodgkin's Lymphoma
}

\author{
Thuanny Silva de Macêdo ${ }^{1 *}$, Maria Cecília Freire de Melo $^{1}$, Juliana Oliveira Vieira ${ }^{2}$ and \\ Aurora Karla de Lacerda Vidal ${ }^{3}$ \\ ${ }^{1}$ Residency in Hospital Dentistry with a focus on Oncology from the University of Pernambuco - ICB / HUOC / UPE, \\ Master student of the Post-Graduation Program in Dentistry, Federal University of Pernambuco - UFPE. \\ ${ }^{2}$ Assistant physician of the Hematology Department of Oswaldo Cruz University Hospital - CEON / HUOC / UPE.
}

\begin{abstract}
${ }^{3}$ Profa. Dra. Adjunct of the General Pathological Processes Discipline of the Institute of Biological Sciences of the University of Pernambuco - ICB / UPE. Head of the Dentistry Department of the Oncology Center of the Oswaldo Cruz University Hospital of the University of Pernambuco - CEON / HUOC / UPE, Coordinator of the Residency Program in Hospital Dentistry with focus on Oncology at the University of Pernambuco - ICB / HUOC / UPE.
\end{abstract}

Received: August 22, 2018; Accepted: September 14, 2018; Published: September 17, 2018

*Corresponding author: Thuanny Silva de Macêdo, Dental Surgeon; Master Student; Federal University of Pernambuco Moraes Rego Avenue, 1235, Recife, Pernambuco, Brazil. ZipCode: 50670-901, Phone: +55 81 996575061; E-mail: thuannymacedo16@gmail.com

\begin{abstract}
Aims: To report a case of female patient with a rare and large extramedullary plasmacytoma in oral cavity.

Methods and Results: This case report refers to a 39-year-old female patient, with a bilateral tissue growth in the region of the lower premolars teeth, presenting reddish color, softened consistency, sessile base and no pain symptomatology. From the clinical data, a diagnostic hypothesis of oral plasmacytoma was reached. The samples collected from the patient underwent histopathological and immunohistochemical analysis for diagnostic purposes. Confirmation of the initial diagnosis of Oral Plasmacytoma was obtained, and bone marrow analysis led the medical team to close a diagnosis of Lymphoplasmacytic Non-Hodgkin's Lymphoma. The treatment of choice was chemotherapy, using the EPOCH regimen. The oral tumors responded well to the chemotherapy regimen, but the patient died due to the systemic condition.
\end{abstract}

Conclusions: Plasmacytomas are malignant and rare tumors of plasma cells, being poorly diagnosed in the oral cavity region. So, it is emphasized the importance of the Dentist's awareness for the early diagnosis and correct referral, thus exerting an important role in the identification of oral manifestations resulting from systemic diseases and vice versa.

Keywords: Oral Cancer; Hematology; Rare Disorders; Plasmacytoma

\section{Introduction}

Plasmacyte neoplasms are characterized by monoclonal proliferation of plasma cells with production of immunoglobulin [1]. They may present as a single lesion (solitary plasmacytoma) or multiple lesions (multiple myeloma). Single lesions usually occur in bone (bone plasmacytoma) but may also occur in soft tissues (extramedullary plasmacytoma) [2]. Its etiology is unknown, being infrequent in the jaws $[3,4]$.
The extramedullary plasmacytomas appear outside the bone marrow and the symptoms vary according to the location of the lesion, being, however, more frequent in head and neck topography [1, 5]. Approximately $80 \%$ of these lesions are located in this region, more frequently in the upper aerodigestive tract, but the occurrence of extramedullary plasmacytomas is uncommon and rare, comprising less than $1 \%$ of malignant neoplasms in the head and neck region $[1,3,4,5]$.

The peak incidence is in the 50-60 years of age, being twice as frequent in males $[2,4]$. Some symptoms may appear due to the location of the tumor. Edema is the most common complaint and painful symptomatology can be reported when there is involvement of the underlying bone. Discomfort or pain in the dental element may occur when the lesion involves the region of the dental alveolus [6].

The clinical picture is non-specific and the definitive diagnosis is through biopsy of the lesion and its anatomopathological examination and immunohistochemical analysis. It is imperative to exclude the presence of multifocal lesions or multiple myeloma, since the evolution of extramedullary plasmacytoma to a multiple myeloma is a determinant factor in patient survival [3].

Radiotherapy is effective in the treatment of extramedullary plasmacytoma, presenting high rates of local control, since the tumor is radiosensitive, and surgery is an option for small and localized lesions $[1,7]$. The long-term prognosis is satisfactory, with 5 -year survival rate ranging from 50 to $82 \%$ for solitary lesions [5].

It is important that the Dentist has knowledge and is able to diagnose oral lesions, which can sometimes signal systemic diseases. Thus, early diagnosis combined with rapid and effective treatment can lead to a better prognosis and improve patient 
survival rates [8]. In view of this, this article proposes to report a case of a patient diagnosed with a rare and large oral cavity plasmocytoma.

\section{Case Report}

The patient signed a Free and Informed Consent Form, consenting to the disclosure of her case for academic purposes.

Patient EMS, female, 39 years old, hospitalized at the Oncology Center of the Oswaldo Cruz University Hospital of the University of Pernambuco (CEON / HUOC / UPE) due to pancytopenia and decline of the general clinical condition. When in contact with the dentistry staff of the service, she reported discomfort in the oral cavity, especially during feeding, and difficulty in performing oral hygiene due to a tissue growth in the gingiva. The intra-oral clinical examination revealed a bilateral tissue growth in the region of the lower premolars teeth, presenting reddish color, softened consistency, sessile base, dental mobility associated with injury and no pain symptomatology (Figure 1a, 1b). For diagnostic purposes, of the oral and the systemic conditions, material was collected from the oral lesions and bone marrow of the patient, which underwent histopathological and immunohistochemical analysis. The reports were conclusive for diagnosis of Oral Plasmocitoma (Reports - № 160297 / A16-09845) and Non-
Hodgkin Lymphoplasmocytic Lymphoma (Reports - № 160297 / A16-09845). In the immunohistochemical analysis of the oral lesion, a CD 38+ plasma cell differentiation and monoclonality for Lambda were observed, being these indicators of oral plasmacytoma. Clinical dental procedures were not performed, but dental follow-up was maintained, and the Standard Operational Protocol of Oral Care [9], adapted [10], was instituted for individuals under antineoplastic therapy, being prescribed use of toothbrush with small head and soft bristles, non-abrasive toothpaste, mouthwash with oral solution of sodium bicarbonate (8 / 8h) and mouthwash with chlorhexidine digluconate $0.12 \%$ (12 / 12h). The medical treatment of choice was chemotherapy, using the EPOCH scheme (used drugs: etoposide, prednisone, vincristine, cyclophosphamide and doxorubicin), with a duration of 6 cycles. The systemic condition and the oral tumors (Figure 1c, 1d), which regressed completely after the third cycle of chemotherapy, responded well to the treatment performed. However, after 5 months of completion of medical treatment, the patient returned to the service complaining of difficulty in locomotion, alteration of lower limb sensitivity, signs of active oncologic disease and medullary compression syndrome, but without sign of recurrence of the oral cavity lesions. One month after the relapse of the systemic condition, the patient died.

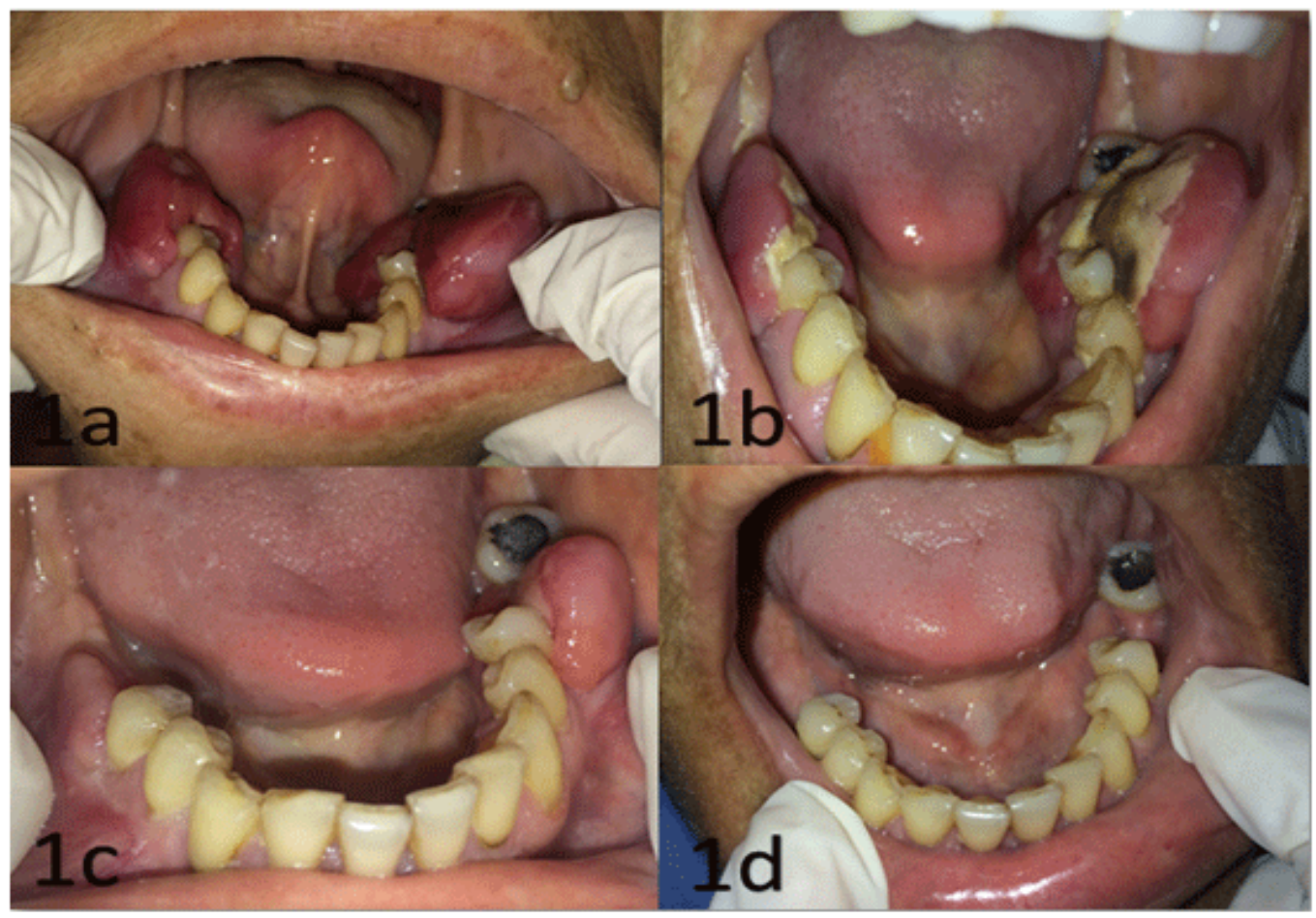

Figure 1: Oral Plasmacytoma: Figure 1a - Initial clinical aspect of the lesion / Figure 1b - Clinical aspect of the lesion during the period of hygiene difficulty / Figure 1c - Clinical aspect of the lesion after the 1st cycle of chemotherapy / Figure 1d - Clinical aspect of the oral cavity after the last cycle of chemotherapy 


\section{Discussion}

Extramedullary plasmacytoma is a rare malignant disease of plasma cells, affecting soft or bone tissue and having multiple myeloma as the main differential diagnosis [4, 7]. More frequent in males and the mean age at diagnosis is 60 years $[2,4,5]$. The case reported in this article differs from the literature, since it affected a young female patient.

The etiology of this condition is still unknown, but chronic stimulation, excessive radiation dose, presence of some types of virus or genetic interactions in the endothelial reticulum system have been pointed out as possible causal factors [3].

Plamacytoma can appear as a circumscribed tumor and it can be confined to the sub mucosa or present an ulcerated surface. The tumor normally has a firm consistency, which can be sessile or pedunculated, the color ranges from red to gray, and easy bleeding is often observed under manipulation [1]. Several of these characteristics were observed in the reported clinical case.

The literature also shows that clinical manifestations of oral cavity plasmocytoma may consist of pain, dental pain, paraesthesia, tooth mobility, dental migration, hemorrhage and even pathological fracture of the involved bone [11, 12].The patient in question did not report painful symptoms, but it was possible to observe a growth of tissue masses and dental mobility. The patient also had an episode of hemorrhage, due to a traumatic injury during feeding.

The diagnosis of extramedullary plasmacytoma in the oral cavity depends initially on a clinical suspicion. Subsequently, in-depth biopsies should be performed and histopathological examination should preferably be accompanied by an immunohistochemical study, in order to confirm the diagnosis through the positivity of plasma cells to the CD38 marker and concomitant cytoplasmic expression of kappa or lambda light chains $[1,8]$, as verified in the immunohistochemical study of this case report.

As this condition is rare, the initial diagnostic hypothesis may be of other malignant tumors more prevalent in the oral cavity, such as squamous cell carcinoma, since the clinical presentation may be similar [1]. Extramedullary plasmacytomas should be distinguished from nonneoplastic lesions like reactive plasmacytic hyperplasia, plasma cell granuloma, pseudo lymphoma and from other malignancies like hematopoietic neoplasm's, malignant melanoma, an aplastic carcinoma, and metastases [3].

Despite being a lesion with a slow and asymptomatic growth, the plasmacytoma can take on large volumes, making appropriate treatment more difficult. When diagnosed early and when there is no bone involvement, treatment success is usually greater. The treatment of choice is radiotherapy, which leads to good results in relation to the remission of the lesion [6]. The option for surgical resection can be considered in case of small lesions or for correction of functional or aesthetic defects. The role of adjuvant chemotherapy is not clearly defined yet, as this type of treatment does not show a decrease in recurrence rates or an increase in the survival rate when compared to isolated radiotherapy [3].
However, chemotherapy treatment should be instituted in cases of disseminated disease [1,3]. In the case in question, since the patient was also diagnosed with another malignant neoplasm, a chemotherapeutic protocol was instituted to control the two conditions.

As previously reported, the patient received a diagnosis of Non-Hodgkin's Lymphoplasmacytic Lymphoma and this condition should also be considered as a differential diagnosis for the lesion present in the oral cavity. This is due to the fact that approximately one third of cases of Non-Hodgkin's Lymphoma occur outside the lymph nodes. Lymphomas are the third most common type of malignancy in the oral cavity, losing only to squamous cell carcinoma and salivary gland neoplasms [13].

Local volume increase, elastic-like mass formation, ulcerations, dental mobility, skin lesions and pain are often reported as symptoms in cases of Non-Hodgkin's Lymphoma in the oral cavity $[14,15]$. These clinical features may be confused with the clinical presentation of oral plasmacytoma, as can be observed in the case in question, then diagnosis of these conditions can be challenging, and when not done according to the recommended methods, can lead to errors and delays in the beginning of treatment [13].

Performing a careful clinical examination of the oral cavity and the entire head and neck region allows the early identification of suspect lesions. Although the treatment of neoplasia belongs to hematologists and oncologists, the dental surgeon plays a fundamental role in the diagnosis of oral cavity lesions [13]. Therefore, well-founded doubts are essential to the diagnosis of oral plasmacytoma, avoiding delays in the diagnostic procedure that consequently lead to a decrease in survival rates [15].

In view of all this, it is emphasized the importance of the Dentist's awareness for the early diagnosis and correct referral, thus exerting an important role in the identification of oral manifestations resulting from systemic diseases and vice versa. Thus, it is essential to recognize changes in bone and oral mucosa patterns, making early diagnosis possible, adequate treatment in a timely manner and better prognosis [8].

\section{Conclusion}

Plasmacytomas are malignant and rare tumors of plasma cells, being poorly diagnosed in the oral cavity region. The literature on the subject is scarce and there are few published cases of oral plasmacytomas. In spite of this, it is extremely important that healthcare professionals consider plasmacytoma as a differential diagnosis, since early diagnosis can lead to an improvement in quality of life, a more adequate therapeutic course and an increase in patient survival.

\section{Declarations}

\section{Conflict of Interest}

The authors declare that there is no conflict of interest

\section{Ethical Approval}

All procedures performed in studies involving human 
participants were in accordance with the ethical standards of the institutional and/or national research committee and with the 1964 Helsinki declaration and its later amendments or comparable ethical standards

\section{Informed consent}

Informed consent was obtained from all individual participants included in the study

\section{References}

1. Corvo MAA, Granato L, Ikeda F, Próspero JD. Extramedullary nasal plasmacytoma: Literature review and a rare case report. Int Arch Otorhinolaryngol. 2013;17(2):213-217.

2. Attanasio G, Viccaro M, Barbaro M, De Seta E, Filipo R. Extramedullary plasmacytoma of paranasal sinuses. A combined therapeutic strategy. Acta Otorhinolaryngol Ital. 2006;26(2):118-120.

3. Allegra A, Nastro Siniscalchi E, Cicciù M, Bacci F, Catalfamo L, Innao V, De Ponte FS, Musolino C, et al. Extramedullary Plasmacytoma of the Maxilla Simulating a Maxillary Radicular Cyst: Quick Diagnosis and Management. J Craniofac Surg. 2016;27(3):296-297. doi: 10.1097/ SCS.0000000000002539

4. Nie S, Peng DC, Gong HH, Ye CL, Nie X, Li HJ. Primary pulmonary plasmacytoma: a case report introduction.World J Surg Oncol. 2016;14:205-210. doi: 10.1186/s12957-016-0948-8

5. Öztürk K, Mustafa S, Midilli R, Gürsan G, Özsan N, Savas R. Extramedullary Plasmacytoma of Head and Neck Region: Report of Six Cases with Different Localizations. Otorhinolaryngology Clinics: An International Journal. 2013;5(3):157-163.

6. Barros TP, Sevilha FM, Amantea DV, Campolongo GD, Neto LB, Alves $\mathrm{N}$, Oliveira RJ, et al. Plamacytoma in the oral cavity: a case report. Int J Odontostomat. 2011;5(2):115-118.
7. Pinto JA, Sônego TB, Artico MS, Leal CFA, Belloto S. Extramedullary plasmacytoma of the larynx. Int Arch Otorhinolaryngol. 2012;16 (3):410-413. doi: 10.7162/S1809-97772012000300019

8. Lúcio PSC, Peixoto TS, Nonaka CFW, Alves PM, Godoy GP. Plasmocitoma solitário do osso: relato e discussão de um caso de acometimento incomum. Rev Assoc Paul Cir Dent. 2015;69(2):165-168.

9. Brasil. Ministério da Saúde. Secretaria de Atenção à Saúde. Instituto Nacional do Câncer. Serviço de Odontologia em Oncologia. Rotinas Internas do INCA. $2^{\mathrm{a}}$ edição rev. amp. Rio de Janeiro: INCA, 2009.

10.Vidal AKL. Protocolo Operacional Padrão de Cuidados Buco-Dentais (POP- Oral) para indivíduos sob terapia antineoplásica. Adaptado das Rotinas Internas do INCA (serviço de Odontologia). 2009.

11. Moshref M, Abbass FM, Sargolzaei S, Nafarzadeh S. Extramedullary plasmacytoma of the gingiva. Arch Iranian Med. 2007;10(1):91-93.

12. Ozdemir R, Kayiran O, Oruc M, Karaaslan O, Kocer U, Ogun D. Plasmacytoma of the hard palate. J Craniofac Surg. 2005;16(1):164 169.

13. Triantafillidou K, Dimitrakopoulos J, Iordanidis F, Gkagkalis A. Extranodal non-hodgkin lymphomas of the oral cavity and maxillofacial region: a clinical study of 58 cases and review of the literature. J Oral Maxillofac Surg. 2012;70(12):2776-2785. doi: 10.1016/j.joms.2012.01.018

14. Deng D, Wang Y, Liu W, Qian Y. Oral and maxillofacial non-Hodgkin lymphomas: Casereportwithreview of literature. Medicine(Baltimore). 2017;96(35):7890-7899. doi: 10.1097/MD.0000000000007890

15. Sirsath NT, Lakshmaiah KC, Das U, Lokanatha D, Chennagiri SP, Ramarao C. Primary extranodal non-Hodgkin's lymphoma of oral cavity--a single centre retrospective study. J Cancer Res Ther. 2014;10(4):945950. doi: 10.4103/0973-1482.136024 